\title{
Interval Based Network Operation Respecting N-1 Security Criterion
}

\author{
P. Voráč, Eduard Janeček, and D. Georgiev
}

\begin{abstract}
Rising penetration of renewable energy sources in present day transmission systems requires increased attention to network security. Existing computation tools in power system operations evaluate individual scenarios for power injection and network configuration but do not fully consider nearby regions in the operating space. Such tools may lead to market transactions, preventive actions, or corrective actions that are nominally efficient but poor in general. In this paper, the interval based ISI method is reformulated to a security oriented form potentially applicable in energy management systems. The presented results include an algorithm defined within a tractable optimization framework that computes maximal power injection sets containing power injection profiles that are necessarily secure in terms of physical constraints and the $\mathrm{N}-1$ security criterion. The method is tested on the IEEE 14 bus test system.
\end{abstract}

\section{INTRODUCTION}

Present day transmission systems in the European Union are operated closer to their security limits. In part this mode of operation is a result of unforeseen power transfers caused by massive installations of renewable energy. In the global effort to curb carbon emissions, governments have proposed many ambitious renewable energy utilisation targets [1], [2], [3] leading to installations of intermittent energy sources at geographical locations with promising energy potential but low energy demand. As a consequence, there is a greater need to evaluate risk associated with operational actions that may violate the network security limits.

For instance, current ancillary service mechanisms used by TSOs are designed to purchase sufficient energy to makeup for any forecasted imbalance between generation and demand at the lowest price regardless of its origin [4]. Such transactions may lead to scenarios where reserved energy cannot be deployed before changing the system operational point through additional redispatch or reconfiguration.

Preventive and corrective redispatch and reconfiguration are yet another opportunity for potentially risky operations. In pursuit of most efficient system settings, various optimal power flow tools exist that propose changes in the system operating point either through reconfiguration or redispatch [5], [6]. Such tools often propose only the final settings and ignore transitions.

Manuscript received May 2, 2014

This work was supported by the grant BIOZE-TA-ČR TA01020865 and by the European Regional Development Fund (ERDF), project "NTIS New Technologies for the Information Society", European Centre of Excellence, CZ.1.1.00/02.0090.

Přemysl Voráč is with the European Development Fund project NTIS, European Centre of Excellence, University of West Bohemia in Pilsen. Daniel Georgiev and Eduard Janeček are with the Department of Cybernetics in the Faculty of Applied Sciences, University of West Bohemia in Pilsen, Pilsen 30614, Czech Republic. vorac@ntis.zcu.cz, georgievekky.zcu.cz
Lastly, operation support tools often fail to incorporate security margins into the optimisation process yielding optimal but fragile states. In other words, the final state may satisfy security criteria but the operator has no idea by what margin.

In general, market and power flow optimisation tools are of the what if type. Optimal power flow tools used to clear intraday and spot electricity markets use simple models to price individual dispatch scenarios but fail to consider actual system safety, availability of regulation, and flexibility of corrective actions [7], [8]. Price sensitivities may be computed using the AC [9] or the approximate DC network model [10] but the relevance to the security of the actual system is unclear. Security constrained optimal power flow tools used to propose reconfiguration and redispatch actions in order to increase system safety also compare individual scenarios but fail to consider nearby regions in the operating space [11]. Stochastic load flow tools based on analytical probabilistic models or point estimate models can be used to investigate consequences of deviations from the proposed operating points [12], [13]. However, the complexity of these tools makes their integration into optimisation frameworks difficult.

Herein the foundations of a security constrained method are introduced. Given a general definition of network security, the method computes maximal interval sets of power injections that are secure in terms of physical constraints (N0 security) as well as in terms of the ssibility of any single line $e_{\lambda}$ the system ( $\mathrm{N}-1$ security). Interval sets are chosen for computational and practical reasons. In practice, the computed interval sets define injection limits, within which redispatch may be performed without the need for coordination. In real world applications, coordination of power system elements is possible with limited precision (due to unbundling of energy provides, using SCADA/EMSs [14].

The method is formulated in terms of deviations from the nominal voltage, hence, even though the resulting sets are a conservative estimate, the suboptimality is negligible [15]. The method algorithms are also based in a tractable optimisation framework suggesting its scalability and implementation robustness may be sufficient enough for market and operational deployment.

Other set based tools that compute power injection security regions exist [16]. However, they do not in general guarantee system security. The underlying models are based

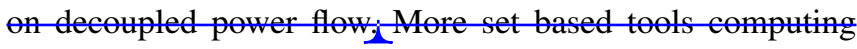
power injection intervals were published recently [17],[18]. However, they consider only tree networks. In computing Available transfer capability and it's sensitivity, set based tools are also used [19], [20]. These tools, however, are not 
optimisation based. Instead, the boundaries of the operating region are approximated by their gradients. This can yield injections violating network security. Furthermore, combinatorial complexity typically associated with tracing boundaries suggests the methods are limited to modest sized problems.

The remainder of the paper is organised as follows. In order to introduce the interval notation, a network model is given in Section III. The method for computing the interval of secure injections is then presented in Section IV. First, the general problem, which is too complex to solve but provides intuition regarding the end goals, is defined. Then, a simplified problem and its approximate solution algorithm are presented. Section $\mathrm{V}$ demonstrates and validates the results through a case study. The paper concludes with Section VI.

\section{NOMENCLATURE}

$\mathcal{N}:$ the set of nodes,

$\mathcal{L}$ : set nodes with uncontrollable injections,

G: set of nodes with controllable injections,

$\mathcal{B}$ : set of branches,

$\mathcal{T}$ : set of network topologies,

$Y_{\tau}$ : admittance matrix,

$X=(\operatorname{Re}(V), \operatorname{Im}(V))=x_{0}+\Delta$ : nodal voltages,

$\chi_{S, \tau}$ : set of secure voltages,

$Z=(P, Q)=\bar{T}(\bar{Z}+W):$ power injections,

$\bar{T}$ : rotational matrix,

$\bar{Z}=z_{0}+A \Delta$ : linear injections,

$z_{S}:$ set of secure injections,

$S$ : branch power flows.

\section{NETWORK MODEL}

\section{A. Notation}

The following notation is used throughout. Capital letters are used to denote matrices and vector variables. Lower case letters are reserved for constants and parameter vectors. Script letters are reserved for sets. Any vector $x \in \mathbb{R}^{n}$ can be written as $\left(x_{1}, \ldots, x_{n}\right)$, as $\left(x_{k}\right)_{k \in\{1, \ldots, n\}}$, or as $x_{\{1, \ldots, n\}}$. The set of real numbers is denoted by $\mathbb{R}$ and the set of complex numbers is denoted by $\mathbb{C}$. Real and imaginary parts of a complex vector $y$ are given by $\operatorname{Re}(y)$ and $\operatorname{Im}(y)$, respectively. The letter $i$ is reserved for the imaginary unit. Transpose and complex conjugates of a complex vector $y$ are given by $y^{T}$ and $y^{*}$, respectively. The absolute value of a complex number $y$ is given by $|y|$ and the $p$-norm of a vector $x$ is given by $\|x\|_{p}$. For a set $X$, the volume of the set is given by $\mu(X)=\int_{X} d x$ and, for two sets $x_{1}, x_{2} \subseteq x$ and mappings $T_{1}, T_{2}: x \rightarrow y, T_{1} x_{1}+$ $T_{2} X_{2}=\left\{y \in y \mid y=T_{1} x_{1}+T_{2} x_{2}, x_{1} \in X_{1}, x_{2} \in X_{2}\right\}$. An n-dimensional closed interval, a cartesian product of 1dimensional closed intervals from $x_{k}^{-}$to $x_{k}^{+}$, is denoted by $\left[x_{k}^{-}, x_{k}^{+}\right]$.

\section{B. Network parameters}

The network is described by a directed graph where each node harbours a potential load or a generator unit and each branch corresponds to a power line or a transformer. The set of nodes is given by the finite set $\mathcal{N}=1, \ldots, n$ and the set of branches is defined by the set $\mathcal{B} \subset \mathcal{N} \times \mathcal{N}$. Possible network topologies are given by the finite set $\mathcal{T}=0,1, \ldots, m$. For a given $\tau \in \mathcal{T}, \mathcal{B}_{\tau} \subseteq \mathcal{B}$. The index $0 \in \mathcal{T}$ denotes the nominal network topology. Node 1 is reserved for the slack bus, where the voltage is held constant and the injected power is adjusted to meet the network demand. The set of nodes with controllable injections (e.g. generators providing ancillary services) but excluding the slack is denoted by $\mathcal{G} \subset \mathcal{N}$ and has the cardinality $g$. The set of nodes with uncontrollable injections (e.g., standard loads or renewable energy sources) is denoted by $\mathcal{L} \subseteq \mathcal{N}$ and has the cardinality $\ell$. The two sets $\mathcal{G}$ and $\mathcal{L}$ satisfy $\mathcal{L} \cap \mathcal{G}=\emptyset$ and $\mathcal{L} \cup \mathcal{G}=\mathcal{N} \backslash\{1\}$. The network admittance matrix for topology $\tau \in \mathcal{T}$ is denoted by $Y_{\tau} \in \mathbb{C}^{n \times n}$.

\section{Network variables}

It is assumed the network is operating under normal conditions under which the single phase model is applicable. Each node $k \in \mathcal{N}$ is associated with a voltage $V_{k} \in \mathbb{C}$ and an injected power $P_{k}+i Q_{k}$. It is often more convenient to list the real and imaginary parts of $V$ separately in a real vector $X=(\operatorname{Re}(V), \operatorname{Im}(V))$ and the real and imaginary parts of the injected powers in a real vector $Z=(P, Q)$. Each branch $b \in \mathcal{B}$ is associated with a power flow $I_{b} \in \mathbb{C}$.

Distinction is made between free injections (those that are not controllable) and controllable injections. Uncontrollable injections at nodes in $\mathcal{L}$ are assumed to be contained in a known set

$$
z_{L}=\left\{\left(P_{\mathcal{L}}, Q_{\mathcal{L}}\right) \mid\left(P_{k}, Q_{k}\right) \in T_{k}\left[z_{k}^{-}, z_{k}^{+}\right], k \in \mathcal{L}\right\},
$$

where the bounds $z_{k}^{-}, z_{k}^{+} \in \mathbb{R}^{2}$ as well as the orthonormal matrix $T_{k} \in \mathbb{R}^{2 \times 2}$ are known.

Definition 1 (Nominal Operating Point): The network has a nominal operating point $x_{0}$ representing the expected network state for the planning horizon. The realised state at the end of this horizon is defined in terms of deviations from this operating point, $X=x_{0}+\Delta$. By the definition of the slack bus, $\Delta_{1}=\Delta_{n}=0$.

Definition 2 (Network Security Domain): Let the state of the network be described by $X=(\operatorname{Re}(V), \operatorname{Im}(V))$. The network security domain for a given topology $\tau \in \mathcal{T}$ is the set $X_{S, \tau} \subseteq \mathbb{R}^{2 n}$ such that, for all $X \in X_{S, \tau}$, the network satisfies the following physical constraints:

$$
\begin{aligned}
& v_{k}^{-} \leq\left|V_{k}\right| \leq v_{k}^{+}, \forall k \in \mathcal{N}, \\
& \left|I_{b}\right| \leq i_{b}^{+}, \forall b \in \mathcal{B}_{\tau} .
\end{aligned}
$$

The exact formulation of the network security domain is not important for the results of this paper. Hence, the above defined conditions can be easily expanded to account for other physical constrains or contingency scenarios. Power injection limits are also omitted as they are part of the problem formulation. 


\section{OPTIMIZATION}

Power injections, for all $\tau \in \mathcal{T}$ and $k \in \mathcal{N}$, are computed from $X$ by the formula [21]

$$
\begin{gathered}
P_{k}=Z_{k}=X^{T} Y_{\tau, k} X, \\
Q_{k}=Z_{k+n}=X^{T} Y_{\tau, k+n} X,
\end{gathered}
$$

where the matrices $Y_{\tau, k}$ and $Y_{\tau, k+n}$ are defined as

$$
\begin{aligned}
Y_{\tau, k} & =\left(\begin{array}{cc}
e_{k} \operatorname{Re}\left(y_{\tau, k}\right) & -e_{k} \operatorname{Im}\left(y_{\tau, k}\right) \\
e_{k} \operatorname{Im}\left(y_{\tau, k}\right) & e_{k} \operatorname{Re}\left(y_{\tau, k}\right)
\end{array}\right), \\
Y_{\tau, k+n} & =\left(\begin{array}{cc}
-e_{k} \operatorname{Im}\left(y_{\tau, k}\right) & -e_{k} \operatorname{Re}\left(y_{\tau, k}\right) \\
e_{k} \operatorname{Re}\left(y_{\tau, k}\right) & -e_{k} \operatorname{Im}\left(y_{\tau, k}\right)
\end{array}\right),
\end{aligned}
$$

with $\left\{e_{k}\right\}_{k \in \mathcal{N}}$ being the set of standard basis vectors in $\mathbb{R}^{n}$ and $y_{\tau, k}$ being the $k$ th row of the admittance matrix $Y_{\tau}$. Note the matrices are not symmetric. This is intentional for reasons that will be made clear below.

\section{A. General Problem}

The problem addressed in the remainder of the paper is defined next.

Problem 1 (General ISI): For all $\tau \in \mathcal{T}$, consider the network security domain $X_{S, \tau}$ and the set of injections from the free buses $z_{L}$. Find a set of secure injections $z_{G}^{*}$ satisfying

$$
\begin{aligned}
z_{G}^{*} & =\operatorname{argmax} \mu\left(z_{G}\right), \text { subject to } \\
z & =\left\{(P, Q) \mid\left(P_{\mathcal{G}}, Q_{\mathcal{G}}\right) \in z_{G},\left(P_{\mathcal{L}}, Q_{\mathcal{L}}\right) \in z_{L}\right\}, \\
z_{G} & =\left\{\left(P_{\mathcal{G}}, Q_{\mathcal{G}}\right) \mid\left(P_{k}, Q_{k}\right) \in T_{k}\left[z_{k}^{-}, z_{k}^{+}\right], k \in \mathcal{G}\right\}, \\
z & \subseteq\left\{Z \mid \forall \tau \in \mathcal{T}, \exists X_{\tau} \in X_{S, \tau}, Z_{k}=X_{\tau}^{T} Y_{\tau, k} X_{\tau},\right. \\
k & \in\{1, \ldots, 2 n\}\} .
\end{aligned}
$$

The matrices $T_{k}$ are rotation matrices satisfying $T_{k}^{T} T_{k}=I$. The set $z_{S}=\left\{(P, Q) \mid\left(P_{\mathcal{G}}, Q_{\mathcal{G}}\right) \in z_{G}^{*},\left(P_{\mathcal{L}}, Q_{\mathcal{L}}\right) \in z_{L}\right\}$ is referred to as the interval of secure injections.

The input to the general ISI problem is the set of possible network topologies $\mathcal{T}$, the network security domains $X_{S, \tau}$, and the admittance matrices $Y_{\tau}$. The output is the set of secure injections $z_{G}^{*}$, defined by the Cartesian product of intervals $\left[z_{k}^{-}, z_{k}^{+}\right] \subset \mathbb{R}^{2}$, for all $k \in \mathcal{G}$ in the range space of the orthonormal matrix $T_{k}$. Hence, $z_{G}^{*}$ is itself an interval in $\mathbb{R}^{2 g}$. The choice of intervals for the set of secure injections has both computational and practical reasons. Generators may be renewable energy sources, whose power injections are influenced by environmental conditions. Generators may be ancillary service providers or sources re-dispatched in corrective actions, whose operation is limited by complex physical constraints dependent on internal hardware limitations. Coordination of such actions in real power systems is performed by SCADA/EMSs to increase the coordination capability. The intervals are not defined in the standard basis. Instead, they are defined in a rotated coordinate frame to capture potential power factor settings of different devices.

Above, optimization is carried out over the variables $z_{k}^{+}, z_{k}^{-}, T_{k}, k=\{1, \ldots, k\}$. The resulting problem seeks to find the limits of injection in all directions and hence is more general than the well known AC OPF problem with a linear criterion, which seeks to maximise injections projected in a single direction. The AC OPF problem is known to be NP hard [21] suggesting the general ISI problem is not easily solvable. A simpler version of the general problem is formulated below and is solved in Section IV-C.

\section{B. Simplified Problem}

The simplified problem (simply referred to as ISI) is defined next.

Problem 2 (ISI): Solve Problem 1 under the following assumptions:

$\mathrm{A} 1: \mathcal{X}_{S, \tau}=\left\{X_{\tau} \mid X_{\tau}=x_{0, \tau}+\Delta, D_{\tau} \Delta \leq d_{\tau}, \tau \in \mathcal{T}\right\}:$ the network security domain is taken to be a bounded convex polytope, where the slack bus constraint $\Delta_{\{1, n\}}=0$ holds, A2: the transformation mappings $T_{k}, k \in \mathcal{G}$, are fixed,

A3: $Z=\bar{T}\left(z_{\tau, 0}+A_{\tau} \Delta+W_{\tau}\right):$ For each $\tau \in \mathcal{T}$, the injections are expanded around the nominal point $x_{\tau, 0}$ and separated into affine terms (referred to as the linear injections) and purely quadratic terms. The quadratic terms $W_{\tau}$ are treated as a disturbance acting to violate Condition 5 in the definition of Problem 1.

The first main simplification is in A1. In practice, one may consider unions of polytopes covering the actual domain with some level of accuracy and repeat the analysis for each polytope separately. A2 includes the second considerable simplification. Coordination of active and reactive power outputs provided by SCADA/EMSs is commonly performed through power factor settings, whose limits are known. In the case study, the nominal power factor is used to a priori compute the matrices $T_{k}$. A3 includes the final important simplification. For each topology $\tau \in \mathcal{T}$, the power vector is expanded around the nominal point so that $Z_{k}=x_{\tau, 0}^{T} Y_{\tau, k} x_{\tau, 0}+x_{\tau, 0}^{T} Y_{\tau, k} \Delta+x_{\tau, 0}^{T} Y_{\tau, k}^{T} \Delta+\Delta^{T} Y_{\tau, k} \Delta$. The expression is then separated into affine and quadratic terms expressed in the rotated coordinate frame, where $\bar{T}_{\{k, k+n\},\{k, k+n\}}=T_{k}$. The quadratic term is then taken to be a disturbance reducing network security. In practice, $\|\Delta\|_{\infty} \ll 1$ in the per unit scale, implying the quadratic terms are likely to be small. One of the key problems resolved in this paper is finding tight bounds on $\Delta$.

In the next section, an approximate solution of ISI is presented. The solution involves three basic steps, each solving one of the following two subproblems. The first subproblem considers a given set of nodes $\mathcal{C} \subseteq \mathcal{N}$ and sets out to find limits on the injections $Z$ for each $\tau \in \mathcal{T}$ with the disturbance $W_{\tau}$ set to zero, i.e., limits on the linear injections $\bar{Z}=z_{\tau, 0}+A_{\tau} \Delta$.

Problem 3 (ISI $\left.I_{1}: \mathcal{C}, \mathcal{T}\right)$ : Consider a set $\mathcal{C} \subset \mathcal{N}$ and the network security domain $X_{S, \tau}$ described by the pair $\left(D_{\tau}, d_{\tau}\right)$. Suppose the parameters $\bar{z}_{k}^{-}$and $\bar{z}_{k}^{+}$are given for all $k \in \mathcal{N} \backslash \mathcal{C}$. Then find the limits $\bar{z}_{\mathcal{C}}^{-, *}, \bar{z}_{\mathcal{C}}^{+, *}$ that solve the following optimisation problem:

$$
\max _{\bar{z}_{\mathcal{C}}^{-}, \bar{z}_{\mathcal{C}}^{+}, \bar{\lambda}_{\mathcal{T}}^{+}, \bar{\lambda}_{\mathcal{T}}^{-}} \prod_{k \in \mathcal{C}} \mu\left(\left[\bar{z}_{k}^{-}, \bar{z}_{k}^{+}\right]\right)
$$


subject to, for all $\tau \in \mathcal{T}, \mathcal{D}_{Z, \tau} \subseteq \mathcal{D}_{\Delta, \tau}$, where

$$
\begin{aligned}
\mathcal{D}_{Z, \tau} & =\left\{\Delta \mid \bar{\lambda}_{\tau}^{-} \leq z_{\tau, 0}+A_{\tau} \Delta \leq \bar{\lambda}_{\tau}^{+}\right\} \\
\mathcal{D}_{\Delta, \tau} & =\left\{\Delta \mid D_{\tau} \Delta \leq d_{\tau}\right\} \\
\bar{\lambda}_{\tau_{0}}^{-} & \leq z_{\tau_{0}, 0} \leq \bar{\lambda}_{\tau_{0}}^{+} \\
\bar{z}^{-} & \geq \bar{\lambda}_{\tau}^{-} \\
\bar{z}^{+} & \leq \bar{\lambda}_{\tau}^{+} \\
\bar{\lambda}_{\tau}^{+} & \geq \bar{\lambda}_{\tau}^{-}
\end{aligned}
$$

where $\left[\bar{\lambda}_{\tau}^{-}, \bar{\lambda}_{\tau}^{+}\right]$represents an injection interval for a single topology $\tau \in \mathcal{T}$. The inequality 8 is optional enabling secure redispatch to the interval of secure injections. ISI $_{1}$ is not trivial if the injection intervals are generalised to polytopes. The problem is then equivalent to finding a maximal polytope embedded in another polytope. Solution to such a problem requires using an exponentially increasing number of constraints.

The second subproblem considers a topology $\tau \in \mathcal{T}$, a convex bounded polytope $\mathcal{D}$ together with a set of nodes $\mathcal{C}$ and sets out to find limits on the quadratic terms $W_{\tau}$.

Problem $4\left(I S I_{2}: \mathcal{C}, \mathcal{D}, \mathcal{T}\right)$ : Consider a node $k \subseteq \mathcal{N}, \tau \in$ $\mathcal{T}$ and a convex bounded polytope $\mathcal{D}$. Find the limits $w_{\tau, k}^{-, *}$ and $w_{\tau, k}^{+, *}$ that solve the following optimisation problem:

$$
\begin{aligned}
& \min \mu\left(\left[w_{\tau,\{k, k+n\}}^{-}, w_{\tau,\{k, k+n\}}^{+}\right]\right) \text {subject to } \\
& W_{\tau,\{k, k+n\}} \in\left[w_{\tau,\{k, k+n\}}^{-}, w_{\tau,\{k, k+n\}}^{+}\right], \forall \Delta \in \mathcal{D} .
\end{aligned}
$$

The structure of the matrices $Y_{\tau, k}$ is used in the next section to approximate the bounds. Finding the exact bounds requires maximising a nonconvex quadratic function over a polytope, an NP hard problem.

\section{Solution}

The ISI solution algorithm presented in this section is outlined in Figure 1. The individual steps as well as some useful facts are described next.

1) Compute bounds on $\bar{Z}_{\{k, k+n\}}, k \in \mathcal{L}$ : One of the inputs is the set of injections from the free buses $Z_{L}$. This set constraints the possible values of $\Delta$ through Equation 2 . These constrains, however, are not amenable to efficient optimisation methods. Hence, the first step is to map the bounds on the injections $Z_{k}$ into conservative bounds on the linear injections $\bar{Z}_{k}$. This is done by computing the bounds on the quadratic terms $W_{\tau}$ (Problem 4). The bounds are computed for all admissible $\Delta$ in a defined polytope $\mathcal{D}$ which is in this step equal to $\mathcal{D}_{\Delta, \tau}$.

Any number of ways may be used to solve Problem 4. The following fact overapproximates the upper and lower bounds of linear injections produced by the free buses. Alternatively, these bounds could be obtained by solving the dual problem.

Fact 1: For topology $\tau \in \mathcal{T}$, consider a polytope $\mathcal{D}$, and, for any $k \in \mathcal{N}$ and $\ell \in\{k, k+n\}$, the set

$$
\mathcal{D}_{\ell}=\left\{\Delta \mid h_{\ell}^{-} \leq \bar{Y}_{\tau, \ell} \Delta \leq h_{\ell}^{+}\right\} \supseteq \mathcal{D}
$$

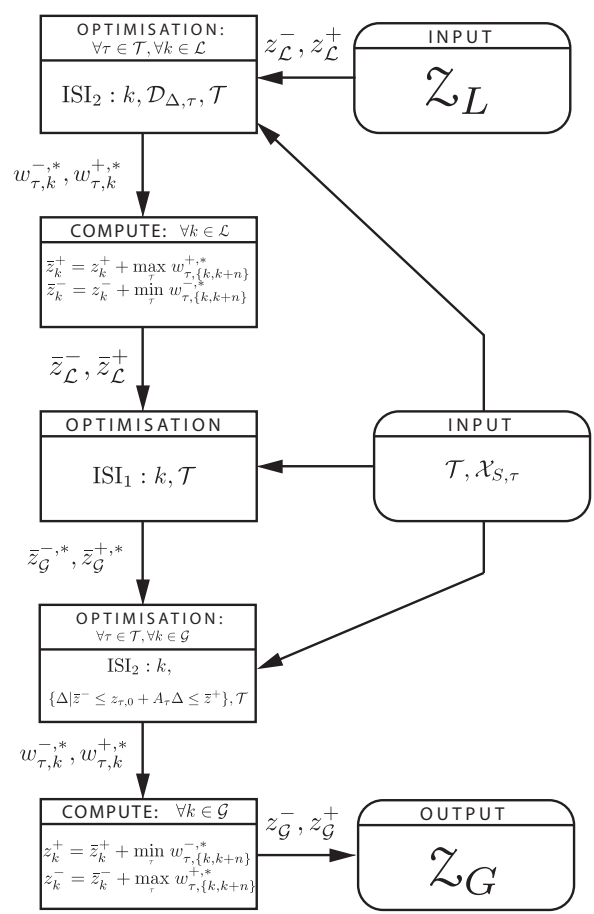

Fig. 1. Flowchart for ISI solution algorithm presented in this paper.

where

$$
\begin{aligned}
\bar{Y}_{\tau, k} & =\left(T_{k}\right)_{1,1}^{-1} Y_{\tau, k}+\left(T_{k}\right)_{1,2}^{-1} Y_{\tau, k+n} \\
\bar{Y}_{\tau, k+n} & =\left(T_{k}\right)_{2,1}^{-1} Y_{\tau, k}+\left(T_{k}\right)_{2,2}^{-1} Y_{\tau, k+n}
\end{aligned}
$$

Then, $\forall \Delta \in \mathcal{D} \cap \mathcal{D}_{\ell}, W_{\tau, \ell}$ is bound by

$$
\begin{aligned}
& W_{\tau, \ell} \geq\left(\Delta_{\{k, k+n\}}^{-}\right)^{T} h_{\ell}^{+}+\left(\Delta_{\{k, k+n\}}^{+}\right)^{T} h_{\ell}^{-} \\
& W_{\tau, \ell} \leq\left(\Delta_{\{k, k+n\}}^{-}\right)^{T} h_{\ell}^{-}+\left(\Delta_{\{k, k+n\}}^{+}\right)^{T} h_{\ell}^{+},
\end{aligned}
$$

where

$$
\begin{aligned}
& \Delta_{\{k, k+n\}}^{-}=\min \left(\Delta_{\{k, k+n\}}, 0\right), \\
& \Delta_{\{k, k+n\}}^{+}=\max \left(\Delta_{\{k, k+n\}}, 0\right) .
\end{aligned}
$$

Computation of the smallest set $\mathcal{D}_{\ell} \supseteq \mathcal{D}$ and the computation of the lower and upper bounds on $W_{\tau, \ell}$ requires solving fixed number of linear programs.

The computed bounds $w_{\tau, k}^{-, *}, w_{\tau, k}^{+*}$ are then used to derive the appropriate bounds on the linear injections

$$
\left[\bar{z}_{, k}^{-, *}, \bar{z}_{k}^{+, *}\right]=\left[z_{k}^{-}, z_{k}^{+}\right]+\left[\min _{\mathcal{T}} w_{\tau,\{k, k+n\}}^{-}, \max _{\mathcal{T}} w_{\tau,\{k, k+n\}}^{+}\right] .
$$

2) Optimise bounds on linear injections for nodes $k \in \mathcal{G}$ and topologies $\tau \in \mathcal{T}$ : The second step is to take known bounds on linear injections and maximise the bounds on the remaining linear injections. This is done by solving $\mathrm{ISI}_{1}$ using the following fact.

Fact 2: Define the submatrices

$$
D_{\tau,-1} \triangleq D_{\tau, \mathcal{N}, \mathcal{N} \backslash\{1\}}, A_{\tau,-1} \triangleq A_{\tau, \mathcal{N} \backslash\{1\}, \mathcal{N} \backslash\{1\}}
$$


and consider the two sets $\mathcal{D}_{Z, \tau}$ and $\mathcal{D}_{\Delta, \tau}$ used in Problem 2. The condition $\mathcal{D}_{Z, \tau} \subseteq \mathcal{D}_{\Delta, \tau}$ holds if and only if

$$
D_{\tau}^{+}\left(\bar{\lambda}_{\tau}^{+}-z_{\tau, 0}\right)+D_{\tau}^{-}\left(\bar{\lambda}_{\tau}^{-}-z_{\tau, 0}\right) \leq d_{\tau},
$$

where

$D_{\tau}^{+}=\max \left\{0, D_{\tau,-1} A_{\tau,-1}^{-1}\right\}, D_{\tau}^{-}=\min \left\{0, D_{\tau,-1} A_{\tau,-1}^{-1}\right\}$

Fact 2 expresses the polytope inclusion constraints in ISI $_{1}$ as linear constraints on the limits $\bar{z}_{\tau}^{-}$and $\bar{z}_{\tau}^{+}$. Subsequently, if we take the logarithm of the utility function, ISI $_{1}$ is converted into a convex optimisation problem. Note, the inverse of $A_{\tau,-1}$ exists if the nominal voltages are different from zero and the network is connected, which is always the case in real world systems.

3) Estimate bounds on quadratic injections for nodes $k \in \mathcal{G}$ and topologies $\tau \in \mathcal{T}$ : The procedure to bound the quadratic terms of the controllable nodes is the same as in Step IV-C.1, with the input polytope $\mathcal{D}$ being $\left\{\Delta \mid \bar{z}^{-} \leq z_{\tau, 0}+A_{\tau} \Delta \leq \bar{z}^{+}\right\}$instead of the larger set $\mathcal{D}_{\Delta, \tau}$. Note, the bounds on all the quadratic terms could have been computed at the outset to yield a greater overapproximation. Similarly, the bounds on the quadratic terms of the free buses could be recomputed to yield a lesser overapproximation.

4) Contract bounds on injections for nodes $k \in \mathcal{G}$ and topologies $\tau \in \mathcal{T}$ : The algorithm terminates once the appropriate bounds on linear injections of controllable nodes are found. The final step is to contract the injection sets $\bar{z}_{\mathcal{G}}^{-, *}, \bar{z}_{\mathcal{G}}^{+, *}$ to obtain conservative bounds on the actual injections. The final bounds are computed as

$$
\left[z_{k}^{-}, z_{k}^{+}\right]=\left[\bar{z}_{k}^{-, *}+\max _{\mathcal{T}} w_{\tau,\{k, k+n\}}^{+}, \bar{z}_{k}^{+, *}+\min _{\mathcal{T}} w_{\tau,\{k, k+n\}}^{-}\right] .
$$

The final bounds $z_{\mathcal{G}}^{-, *}, z_{\mathcal{G}}^{+, *}$ approximate the solution to ISI while ensuring that any injection $Z_{\{k, k+n\}} \in$ $T_{k}\left[z_{k}^{-}, z_{k}^{+}\right], k \in \mathcal{G}$, does not produce an unsafe network state, as long as the injections at the other nodes (free nodes and controllable nodes) are also within their prescribed bounds.

Theorem 1: Consider the set

$$
z_{S}=\left\{(P, Q) \mid\left(P_{k}, Q_{k}\right) \in T_{k}\left[z_{k}^{-}, z_{k}^{+}\right], k \in \mathcal{N}\right\},
$$

where $z_{k}^{-}, z_{k}^{+}$are given for $k \in \mathcal{L}$ and computed by following Steps IV-C.1- IV-C.4 for $k \in \mathcal{G}$. Then, for each $\tau \in \mathcal{T}$ and for any $Z \in Z_{S}$, the resulting voltage lies in the network security domain $x_{S, \tau}$ defined in Problem 2.

Proof 1: Define the following sets:

$$
\begin{aligned}
& x_{L 1}=\left\{X \mid\left(P_{\mathcal{L}}, Q_{\mathcal{L}}\right) \in \mathcal{Z}_{L}\right\}, \\
& x_{L 2}=\left\{X \mid \bar{Z}_{\{k, k+n\}} \in\left[\bar{z}_{k}^{-}, \bar{z}_{k}^{+}\right], k \in \mathcal{L}\right\}, \\
& x_{G 1}=\left\{X \mid \bar{Z}_{\{k, k+n\}} \in\left[\bar{z}_{k}^{-, * *}, \bar{z}_{k}^{+, *}\right], k \in \mathcal{G}\right\}, \\
& x_{G 2}=\left\{X \mid\left(P_{\mathcal{G}}, Q_{\mathcal{G}}\right) \in Z_{G}\right\} .
\end{aligned}
$$

To prove the theorem, it must be shown that $x_{L 1} \cap x_{G 2} \subseteq$ $x_{S, \tau}$. It follows from Fact 1 and ISI $_{2}$, that $x_{L 2} \supseteq x_{L 1}$ and that $x_{L 2} \cap x_{G 1} \supseteq x_{L 2} \cap x_{G 2}$. Hence, $x_{L 2} \cap x_{G 1} \supseteq$ $x_{L 1} \cap x_{G 2}$. The theorem follows from Fact 2 and ISI 1 since for all $\tau \in \mathcal{T}, X_{L 2} \cap X_{G 1} \subseteq X_{S, \tau}$.

\section{CAse Study}

The purpose of the case study is to show possible applications of the ISI method on widely used IEEE test systems [22]. The applicability and computational tractability of the proposed method was tested on the IEEE 14 bus test system. The topology of the test system is shown in Figure 2. The slack bus is located at node 1 . The set of controllable nodes $\mathcal{G}=\{2,3,6,8\}$ contains nodes with connected generators. The set $\mathcal{L}$ contains nodes with known connected load. Lines $\{(5,6),(4,7),(7,9),(4,9)\}$ represent the transformer lines with their respective tap.

The test system is considered in the p.u. scale with the base of 100 MVA. Power injections, impedances, line flow constraints, transformer taps, and topology are taken from [22], [23]. The line current limits $i_{b}^{+}$, for all $b \in \mathcal{B}$ are for the purposes of the case study approximated by the MVA limits provided in [23]. In order to meet load demands and feasible network throughput for the nominal topology $\tau_{0}$, line $(2,3)$ has increased MVA rating by $20 \%$. All optimisation was performed in Matlab using the tool CVX [24].

In the first part of the case study, the network security domain construction is shown. In the second part, the applicability of ISI as a tool for $\mathrm{N}-1$ security assessment is presented. It is shown that ISI provides a different approach to contingency analysis than the optimal point methods (e.g. SCOPF). The third part shows that ISI can also be used as a tool for certified reconfiguration planning while maintaining $\mathrm{N}-1$ security.

All tests were performed on a PC with Intel(R) Core(TM) i5-2520M CPU @ 2.5GHz and 4GB of RAM.

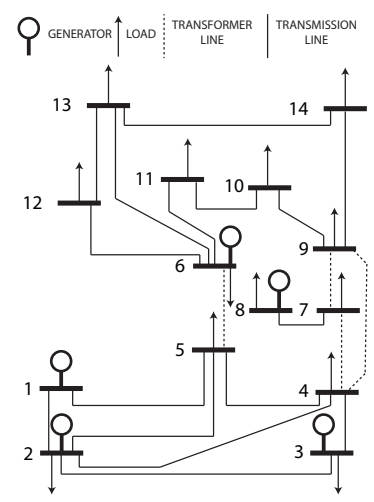

Fig. 2. Test system topology: The IEEE 14 bus test system. The slack bus is located at node 1 . The controllable nodes $\mathcal{G}=\{2,3,6,8\}$ have connected generators. The nodes in $\mathcal{L} \backslash\{1\}$ harbour a given power load. Lines $\{(5,6),(4,7),(7,9),(4,9)\}$ represent the transformer lines. The outage of transformer lines is not considered.

\section{A. Network Security Domain}

The network security domain given in Definition 2 is defined by a convex polytope to satisfy the inequality $D_{\tau} \Delta \leq$ $d_{\tau}$. The matrix $D_{\tau}$ and the vector $d_{\tau}$ contains two types of physical constraints defined next. 


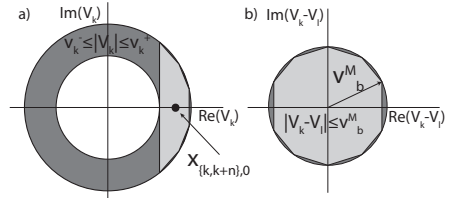

Fig. 3. Illustration of the network security domain: a) The non-convex nodal voltage constraint $v_{k}^{-} \leq\left|V_{k}\right| \leq v_{k}^{+}, \forall k \in \mathcal{N}$ is shown in dark grey. The computed convex polytope is shown in light grey. b) An example of line current constraint represented by a polytope embedded in a circle with radius $v_{b}^{M}$ and center at origin.

1) Nodal voltage constraints: For simplicity, nodal voltage constraints are defined for each node $k \in \mathcal{N}$ as $v_{k}^{-}=$ $0.95\left|x_{\{k, k+n\}}\right|$ and $v_{k}^{+}=1.05\left|x_{\{k, k+n\}}\right|$, i.e., $\pm 5 \%$ of the nominal operating point computed from nominal power injections by the load flow algorithm using the Newton method. This constraint yields a non-convex region; an illustration of the constraint is shown in dark grey in Figure 3 a). Nodal voltage constraints are approximated by an embedded convex polytope also pictured in Figure 3 a) in light grey grey. The nodal constraints for each node $k \in \mathcal{N}$ are defined in the form of matrix inequalities which are then embedded into $D_{\tau}$ and $d_{\tau}$.

2) Line current constraints: Line current constraints $\left|I_{b}\right| \leq i_{b}^{+}$for each branch $b=(k, \ell) \in \mathcal{B}$ can be rewritten to the form

$$
\left|V_{k}-V_{\ell}\right| \leq v_{b}^{M}
$$

where $v_{b}^{M}=i_{b}^{+}\left|Y_{\tau, k, \ell}\right|, \tau \in \mathcal{T}$, denotes the maximum allowed voltage difference. Figure $3 \mathrm{~b}$ ) shows an example of the line current constraint represented by a polytope embedded in a circle with the radius $v_{b}^{M}$ and the center at origin colored in light grey, the approximation error is shown in dark grey. Construction of line current constraints requires to couple nodal voltages of connected nodes. Line current constraints for each branch $b \in \mathcal{B}$ are defined again in the form of matrix inequalities which are embedded into $D_{\tau}$ and $d_{\tau}$.

The final polytope constraint $D_{\tau} \Delta \leq d_{\tau}$ defining the network security domain $X_{S, \tau}$, is defined as an intersection of line current constraints and nodal voltage constraints for each node $k \in \mathcal{N}$ and each branch $b \in \mathcal{B}$.

\section{B. Certified $N-1$ secure injection sets}

In this section, the applicability of ISI as a N-1 security assessment tool is presented. We want to find an interval of secure injections with respect to a given set of topologies $\mathcal{T}$. Topologies are defined in terms of contingency sets $\mathcal{B}_{m_{\tau}}$, where for a topology $\tau \in \mathcal{T}, \mathcal{B}_{\tau}=\mathcal{B} \backslash \mathcal{B}_{m_{\tau}}$. The list of admissible network topologies is given in Table $\mathrm{I}$.

The set $\mathcal{T}$ contains network topologies that yield feasible power flows under nominal power injections and do not affect safe network operation (represented by the converging load flow solution). Transmission line outages creating islands are prevented and transformer line outages are not considered.
TABLE I

CONTINGENCY SETS $\mathcal{B}_{m_{\tau}}$ FOR IEEE 14 BUS TEST SYSTEM

\begin{tabular}{||c||c||c||c||c||c|}
\hline$\tau$ & $\mathcal{B}_{m_{\tau}}$ & $\tau$ & $\mathcal{B}_{m_{\tau}}$ & $\tau$ & $\mathcal{B}_{m_{\tau}}$ \\
\hline 0 & $\emptyset$ & 5 & $\{(3,4)\}$ & 10 & $\{(9,10)\}$ \\
\hline 1 & $\{(1,5)\}$ & 6 & $\{(4,5)\}$ & 11 & $\{(9,14)\}$ \\
\hline 2 & $\{(2,3)\}$ & 7 & $\{(6,11)\}$ & 12 & $\{(10,11)\}$ \\
\hline 3 & $\{(2,4)\}$ & 8 & $\{(6,12)\}$ & 13 & $\{(12,13)\}$ \\
\hline 4 & $\{(2,5)\}$ & 9 & $\{(6,13)\}$ & 14 & $\{(13,14)\}$ \\
\hline
\end{tabular}

Figure 4 shows the solution of ISI with respect to the given set $\mathcal{T}$. The computation time of ISI was $102.3 \mathrm{sec}$. The solution can be interpreted in the following way. Resulting interval of secure injections is guaranteed to be $\mathrm{N}-1$ secure. The redispatch to the $\mathrm{N}-1$ secure interval from the nominal injection point is possible without violation of network physical constraints ( $\mathrm{N}-0$ criterion) since the injection intervals for nominal topology 0 must contain the nominal injection point (Constraint 8).
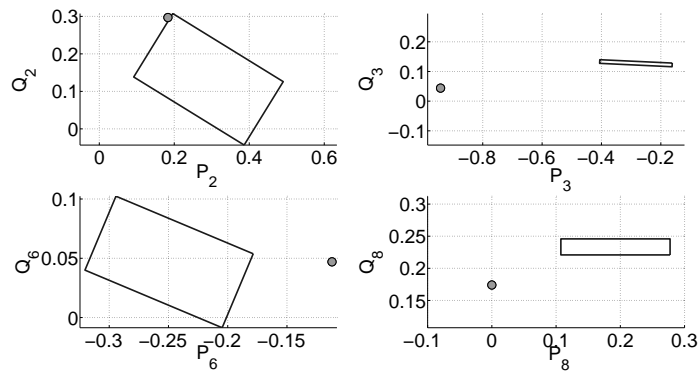

Fig. 4. N-1 secure injection intervals for the nominal topology 0. Nominal power injection points are represented by grey circles. By construction, redispatch from the nominal injection point to interval of secure injections does not violate physical constraints of the system.

This solution yields a greater operational support when compared with the optimal point methods, e.g., SCOPF. The contingency analysis tool also allows the system operator to choose a point from the computed secure injections interval based on other expert knowledge. For instance, the system operator has the possibility to increase system robustness by redispatching the generators closer to the center of the secure injections interval. The increased robustness can protect the system from unexpected power disturbances, e.g., intermittent power injected by renewable energy sources. Additional optimization within the computed intervals (e.g., minimization of economic cost or transmission loss) is easily performed.

\section{C. $N-1$ secure network topology reconfiguration}

In this section, the applicability of ISI as a tool for $\mathrm{N}$ 1 secure network reconfiguration is presented. Suppose the system operator wants to perform a preventive maintenance on line $(10,11)$ of the test system and wants to find the $\mathrm{N}$ 1 secure injections interval for the new topology denoted in Table I by 12 . Hence, the set of topologies $\mathcal{T}$ defined Section $\mathrm{V}-\mathrm{B}$ has to be expanded with the new list of topologies 
TABLE II

SETS $\mathcal{B}_{m_{\tau}}$ ADDED TO $\mathcal{T}$ FOR RECONFIGURATION PLANNING

\begin{tabular}{||c||c||c||c|}
\hline$\tau$ & $\mathcal{B}_{m_{\tau}}$ & $\tau$ & $\mathcal{B}_{m_{\tau}}$ \\
\hline 15 & $\{(1,5),(10,11)\}$ & 20 & $\{(6,12),(10,11)\}$ \\
\hline 16 & $\{(2,3),(10,11)\}$ & 21 & $\{(6,13),(10,11)\}$ \\
\hline 17 & $\{(2,4),(10,11)\}$ & 22 & $\{(9,14),(10,11)\}$ \\
\hline 18 & $\{(2,5),(10,11)\}$ & 23 & $\{(12,13),(10,11)\}$ \\
\hline 19 & $\{(3,4),(10,11)\}$ & 24 & $\{(13,14),(10,11)\}$ \\
\hline 20 & $\{(4,5),(10,11)\}$ & - & - \\
\hline
\end{tabular}

defined by the contingencies listed in Table II yielding a new set $\mathcal{T}_{1}$.

Figure 4 shows the resulting interval of secure injections for $\mathcal{T}_{1}$ in black. The computation time of ISI considering the enhanced set $\mathcal{T}_{1}$ was $194.3 \mathrm{sec}$. The interval of secure injections for $\mathcal{T}$ computed in Section V-B are shown in grey. It can be seen that the intervals of secure injections for $\mathcal{T}$ and $\mathcal{T}_{1}$ are overlapping, i.e., if the power injections at controllable nodes are redispatched to the intersecting intervals, the reconfiguration between topologies 0 and 12 will not require redispatch while maintaining $\mathrm{N}-1$ security. The redispatch to the $\mathrm{N}-1$ secure interval from the nominal injection point is possible without violation of network physical constraints ( $\mathrm{N}-0$ criterion) since the injection intervals for nominal topology 0 must contain the nominal injection point (Constraint 8).
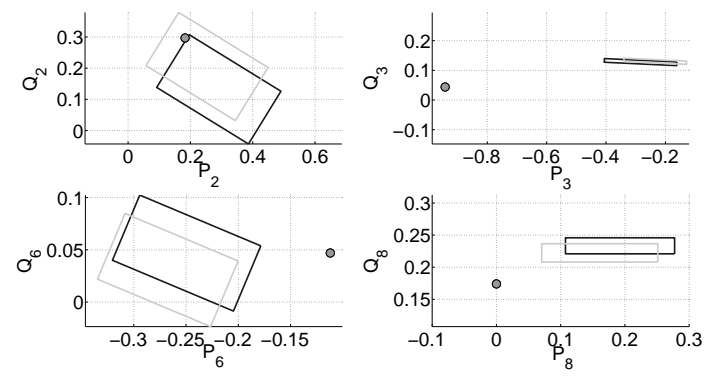

Fig. 5. N-1 secure injections interval considering reconfiguration from topology 0 to topology 12 . Nominal power injection points are represented by grey circles. Resulting interval of secure injections for $\mathcal{T}_{1}$ are plotted in black. The interval of secure injections for $\mathcal{T}$ computed in Section V-B is shown in grey. The intersecting area yields injections where $\mathrm{N}-1$ security holds before and after reconfiguration. It is guaranteed that the redispatch from nominal injection point to the injection intervals does not violate the physical constraints of the system.

\section{CONCLUSION}

A framework and a sufficient solution algorithm for computing interval of secure injections was presented. Given a nominal operating point, the method parametrises a set of injections that necessarily complies with general network security criteria for AC systems as well as with N-1 security criteria. Advantages of ISI compared with known operation support tools (based on point optimization) are potential enhancement of network robustness and broader decision support. The proposed method has a potential to be applied as a real time security management tool allowing N-1 secure network operation and reconfiguration planning.

\section{REFERENCES}

[1] Q. Zhou and J. Bialek, "Generation curtailment to manage voltage constraints in distribution networks," IET Generation, Transmission \& Distribution, vol. 1, 2007.

[2] A. Botterud, J. Wang, C. Moteiro, and V. Miranda, "Wind power forecasting and electricity market operations," in Proc. of the IAEE Int. Conf., 2009.

[3] J. Zhou, "20\% wind generation and the energy markets: A model and simulation of the effect of wind on the optimal energy portfolio," Master's thesis, Princeton University, Dep. of Operations Research and Financial Engineering, 2010.

[4] B. Kirby, "Ancillary services: Technical and commercial insights," Wartsila, Tech. Rep., 2007.

[5] L. Platbrood, H. Crisciu, F. Capitanescu, and L. Wehenkel, "Solving very large-scale security-constrained optimal power flow problems by combining iterative contingency selection and network compression,' in Power system computation conference, 2011.

[6] K. W. Hedman, R. P. O’Neill, E. B. Fisher, and S. S. Oren, "Optimal transmission switching with contingency analysis," IEEE Trans. on Power Systems, 2009.

[7] R. W. Ferrero, S. M. Shahidehpour, and V. C. Ramesh, "Transaction analysis in deregulated power systems using game theory," IEEE Trans. on Power Systems, vol. 12, no. 3, 1997.

[8] E. Litvinov, T. Zheng, G. Rosenwald, and P. Shamsollahi, "Marginal loss modeling in LMP calculation," IEEE Trans. on Power Systems, vol. 19, no. 2, 2004.

[9] H. Liu, L. Tesfatsion, and A. Chowdhury, "Locational marginal pricing basics for restructured wholesale power markets," in Power Energy Society General Meeting, 2009. PES 'O9. IEEE, July 2009, pp. 1-8.

[10] R. Bo and F. Li, "Probabilistic LMP forecasting considering load uncertainty," IEEE Trans. on Power Systems, vol. 24, 2009.

[11] F. Capitanescu, J. L. M. Ramos, P. Panciatici, D. Kirschen, A. M. Marcolini, L. Platbrood, and L. Wehenkel, "State-of-the-art, challenges, and future trends in security constrained optimal power flow," Electrical Power System Research, 2011.

[12] J. M. Morales and J. Perez-Ruiz, "Point estimate schemes to solve the probabilistic power flow," IEEE Trans. on Power Systems., vol. 22, no. 4, 2007.

[13] E. Janeček and D. Georgiev, "Probabilistic extension of the backward/forward load flow analysis method," IEEE Trans. on Power Systems, 2012.

[14] F. Wu, K. Moslehi, and A. Bose, "Power system control centers: Past, present, and future," Proceedings of the IEEE, vol. 93, no. 11, pp. 1890-1908, Nov 2005.

[15] D. Georgiev, E. Janecek, and P. Vorac, "Computing intervals of secure power injection," in to appear in Proc. 19th IFAC World Congress, 2014.

[16] C.-C. Liu, "A new method for the construction of maximal steady-state security regions of power systems," Power Systems, IEEE Transactions on, vol. 1, no. 4, pp. 19-26, Nov 1986.

[17] B. Zhang and D. Tse, "Geometry of injection regions of power networks," Power Systems, IEEE Transactions on, vol. 28, no. 2, pp. 788-797, May 2013.

[18] J. Lavaei, D. Tse, and B. Zhang, "Geometry of power flows and optimization in distribution networks," Power Systems, IEEE Transactions on, vol. 29, no. 2, pp. 572-583, March 2014.

[19] G. Ejebe, J. Tong, G. Waight, J. Frame, X. Wang, and W. Tinney, "Available power transfer capability calculations," IEEE Transactions on Power Systems, vol. 13, no. 4, pp. 1521-1527, 1998.

[20] S. Greene, I. Dobson, and F. L. Alvarado, "Sensitivity of transfer capability margins with a fast formula," IEEE Trans. on Power Systems, 2002.

[21] J. Lavaei and S. H. Low, "Zero duality gap in optimal power flow problem," IEEE Trans. on Power Systems, 2012.

[22] (1993) Power systems test case archive. University of Washington. www.ee.washington.edu/research/pstca.

[23] S. K. M. Kodsi and C. A. Canizares, "Modeling and simulation of IEEE 14 bus system with facts controllers," University of Waterloo, Tech. Rep., 2003.

[24] I. CVX Research, "CVX: Matlab software for disciplined convex programming, version 2.0," http://cvxr.com/cvx, aug 2012. 